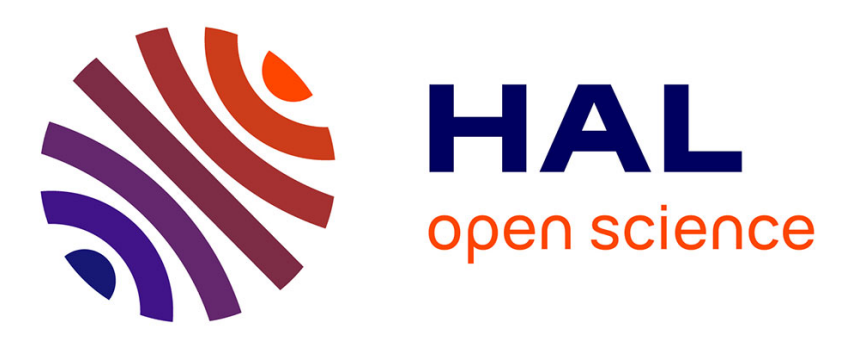

\title{
High levels of uPA and PAI-1 predict a good response to anthracyclines
}

Simona Borstnar, Aleksander Sadikov, Barbara Mozina, Tanja Cufer

\section{To cite this version:}

Simona Borstnar, Aleksander Sadikov, Barbara Mozina, Tanja Cufer. High levels of uPA and PAI-1 predict a good response to anthracyclines. Breast Cancer Research and Treatment, 2009, 121 (3), pp.615-624. 10.1007/s10549-009-0691-8 . hal-00612980

\section{HAL Id: hal-00612980 \\ https://hal.science/hal-00612980}

Submitted on 2 Aug 2011

HAL is a multi-disciplinary open access archive for the deposit and dissemination of scientific research documents, whether they are published or not. The documents may come from teaching and research institutions in France or abroad, or from public or private research centers.
L'archive ouverte pluridisciplinaire HAL, est destinée au dépôt et à la diffusion de documents scientifiques de niveau recherche, publiés ou non, émanant des établissements d'enseignement et de recherche français ou étrangers, des laboratoires publics ou privés. 


\title{
High levels of uPA and PAI-1 predict a good response to anthracyclines
}

\author{
Simona Borstnar • Aleksander Sadikov • \\ Barbara Mozina - Tanja Cufer
}

Received: 6 June 2009/ Accepted: 14 December 2009/Published online: 29 December 2009

(C) Springer Science+Business Media, LLC. 2009

\begin{abstract}
Urokinase-type plasminogen activator (uPA) and its main inhibitor (PAI-1) were shown with level 1 evidence to be prognostic factors for primary breast cancer. Our preliminary retrospective study on a cohort of 1,220 consecutive patients hinted that UPA and PAI-1 could also serve as predictive factors for systemic therapy, namely that patients with high levels of the two markers benefit much more from anthracycline-based chemotherapy than patients with low levels of the two markers. The latter could equally well be treated with less toxic CMF-based chemotherapy (cyclophosphamide, methotrexate, and fluorouracil). The retrospective study, however, suffered from severely uneven patient and tumor characteristics as the patients were treated per institutional guidelines valid at the time and were not randomized between the anthracycline and CMF arms. In the present paper, we attempted to remedy this shortcoming and recheck our previous observations on more balanced data. To this end we employed a custom-made computer algorithm that selected 180 patients out of a total of 1,220 patients such that we obtained very well balanced anthracycline and CMF arms according to patient and tumor characteristics. Moreover, the low and high uPA/PAI-1 subgroups within both arms were also completely balanced. The algorithm in a way
\end{abstract}

S. Borstnar $(\bowtie) \cdot$ B. Mozina

Institute of Oncology, Zaloska 2, Ljubljana 1000, Slovenia

e-mail: sborstnar@onko-i.si

\author{
A. Sadikov \\ Faculty of Computer and Information Science, Artificial \\ Intelligence Laboratory, University of Ljubljana, Trzaska 25, \\ Ljubljana 1000, Slovenia \\ T. Cufer \\ University Clinic Golnik, Golnik 4204, Slovenia
}

created a similar setting to that of a randomized study at the expense of greatly reducing the number of patients included into the study. In this setting, we observed the 3-year disease-free survival (DFS) in all four subgroups (according to treatment and levels of markers: both UPA and PAI-1 low versus one or both high). We report that the 3-year DFS in the CMF arm differed significantly: $87.1 \%$ for patients with low levels of markers versus $77.0 \%$ for patients with high levels of markers $(P=0.044$, $\mathrm{HR}=2.81,95 \% \mathrm{CI}=0.98-8.04)$. On the other hand, the 3 -year DFS in the anthracycline arm did not differ much between the two marker level subgroups: $85.2 \%$ for patients with low levels of markers versus $81.8 \%$ for patients with high levels of markers. Our observation points out that worse prognosis correlated to high uPA and PAI-1 levels can be reversed by treatment efficacy achieved through anthracycline-based chemotherapy. Based on this observation, we hypothesize that uPA/PAI-1 combination could be predictive for response to systemic therapy.

Keywords uPA - PAI-1 - Predictive value . Breast cancer - Anthracycline-based chemotherapy . Balancing patients' and tumor characteristics

\section{Introduction}

The overview of the Early Breast Cancer Trialists' Collaborative Group demonstrates that adjuvant hormonal therapy and polychemotherapy substantially reduce the risk of both recurrence and death from breast cancer and that anthracycline-based chemotherapy is more effective than non-anthracycline, taking into account that the most frequently used non-anthracycline-based chemotherapy in the 
overview trials was CMF [1]. However, since the adjuvant systemic therapy does have associated risks, it would be useful to optimally select the patients who are more likely to respond to and benefit from a certain kind of systemic therapy. Therefore, in the clinical management of breast cancer, the identification of predictors of response to therapy may have an important impact on treatment decision, thereby improving clinical outcome.

A predictive marker can be defined as a factor which predicts response or resistance to a particular therapy. Standard predictive markers include hormone receptor status for response to endocrine therapy and HER-2 amplification and/or overexpression for response to antiHER-2 therapy in both metastatic and adjuvant setting. Recently, HER-2 status was also recognized as a predictive marker for response to anthracycline-containing chemotherapy. In a meta-analysis with 8 large prospective trials included, the significant benefit of anthracycline-containing regimens over non-anthracycline ones was observed in women with HER-2 positive early breast cancer [2]. Preclinical as well as clinical data indicate that the increased anthracycline sensitivity might actually not be due to HER2 amplification/overexpression, but rather to topoisomerase $\mathrm{II} \alpha$ gene $(T O P 2 A)$ amplification or protein overexpression $[3,4]$, the major molecular target of anthracyclines.

Urokinase-type plasminogen activator (uPA) and its main inhibitor (PAI-1) were shown with level 1 evidence to be prognostic factors for primary breast cancer. In numerous individual trials (reviewed [5-8]), in our own preliminary work [9-11], as well as in a pooled analysis of 8,377 breast cancer patients from 18 different data sets [12] uPA and PAI-1 uniformly showed independent prognostic value. Most of the patients included in these early studies did not receive any chemotherapy, while endocrine therapy was performed in a majority of hormonal receptor positive patients. A prospective randomized clinical study Chemo N0 [13, 14] randomizing patients with high levels of uPA or PAI-1 between chemotherapy and no chemotherapy while leaving patients with low levels of UPA and PAI-1 out of any adjuvant systemic therapy established a level 1 prognostic value of these two markers in early breast cancer. This was actually the first prospective randomized trial demonstrating that patients with good prognosis, according to low uPA and PAI-1 levels, could be spared toxic chemotherapy.

The observation that the prognostic value of both uPA and PAI-1 was lost in patients who received the adjuvant therapy observed in Chemo N0 trial [13, 14] as well as in other trials [15-18] resulted in the hypothesis that these two markers might have predictive value for response to systemic therapy as well. The correlation of inherited worse prognosis associated with a particular prognostic factor diminishing with treatment efficacy points out to a possible predictive value of this particular factor. The difference between the prognostic and predictive value of a marker was nicely explained and graphically displayed by Hamilton and Piccart [19].

The findings of different clinical trials which investigated this issue regarding UPA and PAI-1 are not uniform. We reported on a possible predictive value of $\mathrm{UPA} / \mathrm{PAI}-1$ for response to hormonal therapy in a group of 460 breast cancer patients [11]. In our collective of patients treated with tamoxifen, the bad prognosis associated with high levels of uPA/PAI-1 was lost. Similar observations were made by the Munich [15, 16], Swedish [17] and Dutch [18] groups, indicating that high levels of uPA and/or PAI-1 in the primary tumor could predict for a better response to the adjuvant tamoxifen treatment. On the contrary, in a larger data set obtained from the German and Dutch groups, containing 3,424 patients, no significant interactions between hormonal therapy and uPA/PAI-1 levels were found. However, in the frame of this pooled analysis, the observation that the patients with high uPA/PAI-1 levels might have benefited more from the adjuvant chemotherapy than those with low levels of uPA/PAI-1 was made for the first time [20]. In another Dutch study with 1,119 patients included, it was also found that the expression of the UPA:PAI-1 complex independently predicted the efficacy of adjuvant chemotherapy [18]. In the already mentioned prospective randomized Chemo NO trial, high uPA and PAI-1 levels were associated with a greater benefit (i.e. $52 \%$ ) from chemotherapy, namely $\mathrm{CMF}$, than expected according to the overview data $[13,14]$.

Anthracycline-based adjuvant chemotherapy results in significantly (10\%) higher benefit compared to CMF chemotherapy in unselected breast cancer populations according to the overview data. However, serious longterm toxicities such as cardiotoxicity and secondary cancers have been observed in a small proportion of patients treated with anthracyclines. The selection of patients that might benefit from anthracyclines is therefore of utmost importance.

In our collective of early breast cancer patients treated in clinical practice, we have already reported that patients with high uPA and PAI-1 levels in primary tumor might benefit more from the anthracycline-based regimens than from CMF. Our preliminary retrospective observation was based on a cohort of 1,220 consecutive patients, the majority of whom were treated with some kind of adjuvant systemic therapy. Already in this unselected population of patients we observed a significant difference in disease-free survival (DFS) with respect to uPA and PAI-1 levels in the whole cohort of patients and in patients treated by CMF, while the significance was lost in the subgroup of patients treated by anthracycline-containing regimens [21]. The retrospective study, however, suffered from severely 
uneven patient and tumor characteristics as the patients were treated per institutional guidelines valid at the time and were not randomized between the anthracycline and CMF arms. In the present paper, we attempted to remedy this shortcoming and recheck our previous observations on more balanced data. To this end we employed a custommade computer algorithm to select well balanced anthracycline and CMF arms according to patient and tumor characteristics. This retrospective study adheres to REMARK criteria [22].

\section{Patients and methods}

\section{Patients}

From 1997 until 2001, uPA and PAI-1 levels were prospectively determined in tumor extract of 1,220 patients with early breast cancer treated at the Institute of Oncology, Ljubljana. All of the patients were female and had a histologically confirmed invasive breast cancer. The established clinical and histomorphological factors, such as menopausal status, tumor size, nodal status, pathological grade, hormonal receptors and tumor vessel invasion, were determined. Tumor grade was scored according to the ScarffBloom-Richardson classification modified by Elston [23]. Steroid hormone receptors were determined either in cytosol fractions using a biochemical method or by immunohistochemical staging on paraffin-embedded tissue sections. The values equal to or greater than $10 \mathrm{fmol} / \mathrm{mg}$ protein as measured by biochemical method or positive staining in more than $10 \%$ of tumor cells by immunohistochemical method for one or both steroid hormone receptors were considered as positive hormone receptor status.

Treatment decisions regarding primary surgery and adjuvant systemic therapy were based primarily on the guidelines valid at the time and did not take into account either uPA or PAI-1 levels. All patients underwent radical local treatment. Most of the patients were treated with some kind of adjuvant systemic therapy: chemotherapy (ChT, $n=331$ ), hormonal therapy (HT, $n=379$ ), or a combination of both $(n=365)$. Of 696 patients treated with ChT, either alone or in combination with HT, 368 (53\%) received CMF and $328(47 \%)$ were treated with anthracycline-containing regimens (28 patients in combination with taxanes). The values of either uPA or PAI-1 were not determined for 41 of these 696 patients, therefore these patients were excluded from the analysis. After the completion of primary treatment, the patients underwent regular follow-up examinations at our institute. All the procedures were in accordance with the ethical standards of the Medical Ethics Committee at our Institute and with the Helsinki declaration.
Statistical methods

The endpoint in this study was disease-free survival (DFS). It was calculated from the date of the start of primary therapy to the date of breast cancer recurrence (locoregional or distant), the date of death from any cause, or the date of the last follow-up; censored observations correspond to patients alive and without evidence of disease recurrence at the time of the last follow-up. DFS as a function of the markers studied was estimated by the Kaplan-Meier method and the log-rank test was used to test for differences. The Cox multivariate hazards models were used to calculate the hazard ratios (HR) and their $95 \%$ confidence intervals (95\% CI) in the analysis of DFS. Computations were performed with the use of the SPSS 12 statistical package. All reported $P$ values are two-tailed.

\section{Laboratory assays of uPA and PAI-1}

Tumor specimens were obtained from primary tumors and stored in liquid nitrogen until extraction. The frozen tumor tissue samples were pulverized using a micro-dismembrator. The tumor powder was suspended in a buffer $(\mathrm{pH} 8.5)$ containing $0.02 \mathrm{mM}$ Tris- $\mathrm{HCl}, 0.125 \mathrm{mM} \mathrm{NaCl}$ and $2 \%$ Triton X-100, and shaken for $3 \mathrm{~h}$ at $4^{\circ} \mathrm{C}$. The obtained suspension was then centrifuged for $30 \mathrm{~min}$ at $100,00 \times \mathrm{g}$. Protein content was determined by using the Pierce assay. Both biological markers were determined in tumor detergent extracts by commercially available enzyme-linked immunosorbent assays (American Diagnostica Inc., Greenwich, CT). International quality assurance of the kits is guaranteed, and the kits have been assessed by the EORTC RBG [24]. Validated cut-off values were assigned for uPA (3 ng/mg protein) and PAI-1 (14 ng/mg protein). According to the combination of uPA and PAI-1 values, patients were divided into two categories: both factors low (low group) versus one or both factors high (high group).

\section{Balancing the treatment groups}

In the retrospective evaluation of a prognostic and/or predictive factor, the research and the comparator groups are usually unbalanced with respect to other patient and tumor characteristics. The purpose of a matching algorithm is to find such subgroups of the given initial groups as to minimize the differences in the distributions of selected characteristics. The matching algorithms in the literature, surveyed by $\mathrm{Gu}$ and Rosenbaum [25], usually pick tuples of patients in each step-one (or more) belonging to each group that differ less than some prescribed threshold between one another. In their most basic and rigorous form, such algorithms pick only the tuples of patients that are 
identical with respect to all characteristics of interest. The algorithms repeat this selection step for as long as possible.

The main goal of our research was to investigate the potential predictive value of uPA and PAI-1 in treatment groups as well-balanced as possible with respect to standard prognostic factors. Our problem was as follows: we had to match the patients in four observation groups as given in Fig. 1 (CMF low, CMF high, ANT low, and ANT high) with respect to seven characteristics: menopausal status, adjuvant hormonal treatment, tumor grade, tumor size, hormonal receptor status, vascular invasion, and lymph node status. In our case, matching algorithms failed to yield subgroups of sufficiently large sizes. Our problem was specific in the sense that we had to match four groups with severely different distributions of characteristics due to inherent characteristic distributions related to low versus high uPA/PAI-1 levels, as well as due to the bias in chemotherapy selection. One look at Table 1 shows that, in our setting, there simply were not enough tuples of four patients (one from each group) to match completely in every characteristic — there are extreme differences in distributions, especially in lymph node status, vascular invasion, tumor size, and tumor grade.

Since the matching algorithms failed, we decided to match the groups as a whole and not on a patient-by-patient level. However, since matching on the group level is weaker in terms of matching quality, we actually combined the two approaches to get a reasonable compromise between the quality of matching and subgroup sizes. The algorithm was custom-built specifically for our problem and its main ideas were as follows (these ideas, however, are general and can be applied to other difficult cases where matching algorithms fail).

First, we took the two smallest (and thus most problematic) groups, namely CMF low and ANT low, and matched them in two steps. In the first step, we selected the patients with identical characteristics from the two groups (perfect matches). In the second step, we additionally selected patients that differed in at most two characteristics, but not in the lymph node status. Furthermore, in the second step, we selected the new patients only if they did not violate the predescribed maximum difference in the distributions between groups (the difference was measured in terms of the Pearson chi-square statistic). Such a procedure is greedy and myopic in the sense that a subsequently selected patient could repair the imbalance created by the current patient; however, taking that into account would be computationally too ineffective due to combinatorial explosion. This procedure yielded two smallest subgroups (CMF low and ANT low). From the two remaining groups we then selected the patients that matched as closely as possible with the patients in the two already set-up subgroups.

The selection of patients from the two larger groups was slightly different. For each patient from the two smaller subgroups, we selected up to three (if possible) patients from these two groups that best matched it-but never allowed a mismatch in more than two characteristics and never a mismatch in lymph node status. Violation in distribution was not checked in this step as it was found to be too restrictive. Since this procedure yielded the subgroups that violated the maximum predescribed difference in the distributions between subgroups, they had to be trimmed down. We only trimmed the two larger subgroups and did this as follows. If a violation in any of the characteristics of interest remained, we excluded the patient that most contributed to the violation in general (not just in one characteristic), while not making another characteristic violated. The patient that most skewed the distributions was selected by a greedy search with a specially designed heuristic using as its basis the Pearson chi-square statistic. Finally, the patients that remained were grouped in the two remaining subgroups (CMF high and ANT high). The quality of the final match and the subgroup sizes are two contradicting criteria. To get larger subgroup sizes we sacrificed menopause as the least important characteristic.

The success of our balancing algorithm (the term balancing is used here to separate this algorithm from the matching algorithms and because it better describes the idea of the algorithm) can be seen in Table 2. It shows

Fig.1 Experimental design

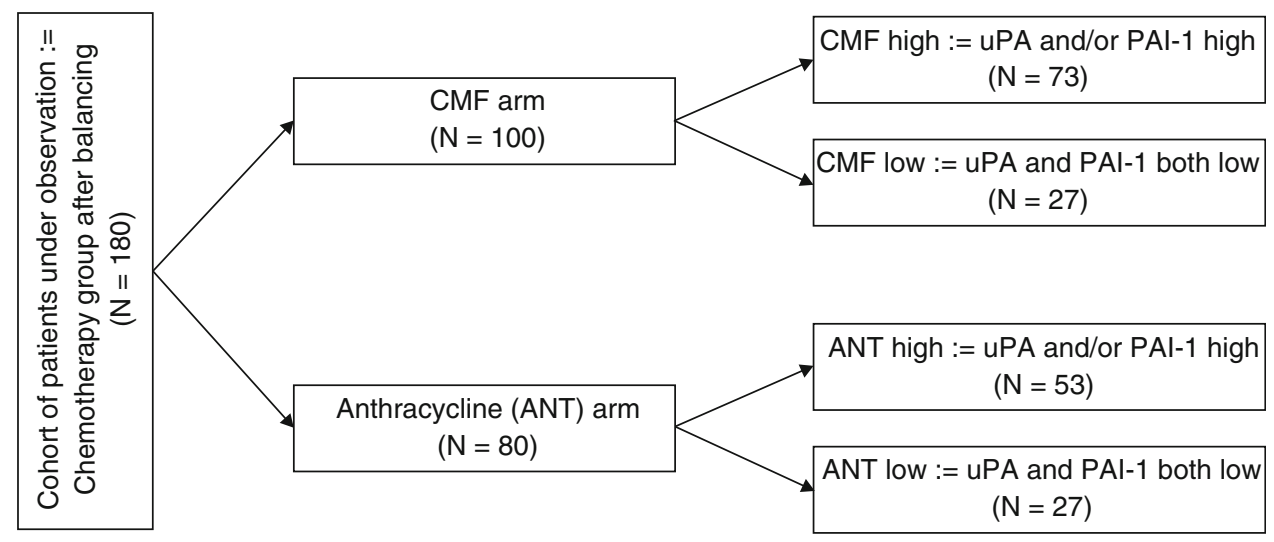


Table 1 Patient and tumor characteristics before balancing

\begin{tabular}{|c|c|c|c|}
\hline Characteristic & $\mathrm{CMF}$ & ANT & $P$ (treatment) \\
\hline \multicolumn{4}{|c|}{$\mathrm{uPA} / \mathrm{PAI}-1$ status $=$ both low } \\
\hline \multicolumn{4}{|c|}{ Menopausal status } \\
\hline Pre/peri & 43 & 36 & 0.340 \\
\hline Post & 36 & 42 & \\
\hline \multicolumn{4}{|c|}{ Adjuvant treatment } \\
\hline ChT only & 25 & 25 & 1.000 \\
\hline ChT \& HT & 55 & 53 & \\
\hline \multicolumn{4}{|l|}{ Tumor grade } \\
\hline Grade I & 11 & 3 & 0.045 \\
\hline Grade II & 43 & 37 & \\
\hline Grade III & 26 & 35 & \\
\hline Unknown & 0 & 3 & \\
\hline \multicolumn{4}{|l|}{ Tumor size } \\
\hline$\leq 20 \mathrm{~mm}$ & 36 & 18 & 0.007 \\
\hline$>20 \mathrm{~mm}$ & 44 & 58 & \\
\hline Unknown & 0 & 2 & \\
\hline \multicolumn{4}{|l|}{ HR status } \\
\hline Negative & 11 & 15 & 0.396 \\
\hline Positive & 69 & 63 & \\
\hline \multicolumn{4}{|c|}{ Vascular invasion } \\
\hline No & 55 & 34 & $<0.001$ \\
\hline Yes & 17 & 40 & \\
\hline Unknown & 8 & 4 & \\
\hline \multicolumn{4}{|c|}{ Lymph node status } \\
\hline Negative & 39 & 2 & $<0.001$ \\
\hline 1-3 positive & 32 & 23 & \\
\hline$>3$ positive & 7 & 53 & \\
\hline Unknown & 2 & 0 & \\
\hline \multicolumn{4}{|c|}{$\mathrm{uPA} / \mathrm{PAI}-1$ status $=$ one or both high } \\
\hline \multicolumn{4}{|c|}{ Menopausal status } \\
\hline Pre/peri & 111 & 105 & 0.013 \\
\hline Post & 169 & 100 & \\
\hline Unknown & 9 & 3 & \\
\hline $\mathrm{p}(\mathrm{uPA} / \mathrm{PAI} 1)^{*}$ & 0.021 & 0.506 & \\
\hline \multicolumn{4}{|c|}{ Adjuvant treatment } \\
\hline ChT only & 168 & 95 & 0.006 \\
\hline ChT \& HT & 121 & 113 & \\
\hline $\mathrm{p}(\mathrm{uPA} / \mathrm{PAI} 1)^{*}$ & $<0.001$ & 0.044 & \\
\hline \multicolumn{4}{|l|}{ Tumor grade } \\
\hline Grade I & 12 & 10 & 0.058 \\
\hline Grade II & 87 & 43 & \\
\hline Grade III & 189 & 155 & \\
\hline Unknown & 1 & 0 & \\
\hline $\mathrm{p}(\mathrm{uPA} / \mathrm{PAI} 1)^{*}$ & $<0.001$ & $<0.001$ & \\
\hline \multicolumn{4}{|l|}{ Tumor size } \\
\hline$\leq 20 \mathrm{~mm}$ & 98 & 55 & 0.076 \\
\hline$>20 \mathrm{~mm}$ & 187 & 152 & \\
\hline Unknown & 4 & 1 & \\
\hline
\end{tabular}

Table 1 continued

\begin{tabular}{llll}
\hline Characteristic & CMF & ANT & $P$ (treatment) \\
\hline p(uPA/PAI1)* & 0.089 & 0.759 & \\
HR status & & & \\
Negative & 97 & 71 & 0.924 \\
Positive & 191 & 137 & \\
Unknown & 1 & 0 & \\
p(uPA/PAI1)* & $<0.001$ & 0.014 & \\
Vascular invasion & & & \\
No & 184 & 96 & \\
Yes & 79 & 103 & \\
Unknown & 26 & 9 & $<0.001$ \\
p(uPA/PAI1)* & 0.307 & 0.786 & \\
Lymph node status & & & \\
Negative & 153 & 23 & \\
1-3 positive & 103 & 60 & \\
$>3$ positive & 32 & 125 & \\
Unknown & 1 & 0 & \\
p(uPA/PAI1)* & 0.658 & 0.072 &
\end{tabular}

Note: Groups were compared using Fisher's exact test where possible, otherwise the Kruskall-Wallis test was used

* These $P$-values (uPA/PAI1) are for the characteristics grouped by treatment, e.g. comparing lymph node status for ANT-treated patients separately from CMF-treated patients with respect to uPA/PAI-1 status

the distributions of the aforementioned characteristics after applying the balancing algorithm. We can see that the groups are now well balanced with respect to the selected characteristics. The characteristic, whose distribution most varies between any two subgroups, is the hormonal receptor status between CMF high and ANT high subgroups-yet, this difference is not so large.

Heuristic (Pearson) thresholds were arbitrarily selected and experimented with to give us the best possible compromise between the number of included cases (arm strength) and similarity of distributions. This experimentation, however, was performed in advance and independently of the data analysis. Only after being satisfied with both the number of included patients and the similarity of distributions within the observed groups did we perform the analysis, not alternating between the two steps to give us the sought after results.

The balancing algorithm is deterministic; the same input data consisting of thresholds and patients with their characteristics always give the same result. The algorithm with final threshold settings was run on lexicographically ordered patients. This enables us to reproduce and verify its results and at the same time helps prevent any possible biases since the lexicographic order of patients does not correlate with any selected or observed characteristic. 
Table 2 Patient and tumor characteristics after balancing

\begin{tabular}{|c|c|c|c|}
\hline Characteristic & CMF & ANT & $P$ (treatment) \\
\hline \multicolumn{4}{|c|}{$\mathrm{uPA} / \mathrm{PAI}-1$ status $=$ both low } \\
\hline \multicolumn{4}{|c|}{ Menopausal status } \\
\hline Pre/peri & 12 & 11 & 1.000 \\
\hline Post & 15 & 16 & \\
\hline \multicolumn{4}{|c|}{ Adjuvant treatment } \\
\hline ChT only & 7 & 6 & 1.000 \\
\hline ChT \& HT & 20 & 21 & \\
\hline \multicolumn{4}{|l|}{ Tumor grade } \\
\hline Grade I & 2 & 2 & 0.958 \\
\hline Grade II & 16 & 15 & \\
\hline Grade III & 9 & 10 & \\
\hline \multicolumn{4}{|l|}{ Tumor size } \\
\hline$\leq 20 \mathrm{~mm}$ & 8 & 6 & 0.757 \\
\hline$>20 \mathrm{~mm}$ & 19 & 21 & \\
\hline \multicolumn{4}{|l|}{ HR status } \\
\hline Negative & 3 & 4 & 1.000 \\
\hline Positive & 24 & 23 & \\
\hline \multicolumn{4}{|l|}{ Vascular invasion } \\
\hline No & 19 & 16 & 0.555 \\
\hline Yes & 7 & 10 & \\
\hline Unknown & 1 & 1 & \\
\hline \multicolumn{4}{|c|}{ Lymph node status } \\
\hline Negative & 2 & 2 & 1.000 \\
\hline 1-3 positive & 17 & 17 & \\
\hline$>3$ positive & 8 & 8 & \\
\hline \multicolumn{4}{|c|}{ uPA/PAI-1 status $=$ one or both high } \\
\hline \multicolumn{4}{|c|}{ Menopausal status } \\
\hline Pre/peri & 27 & 33 & 0.007 \\
\hline Post & 46 & 20 & \\
\hline $\mathrm{p}(\mathrm{uPA} / \mathrm{PAI} 1)^{*}$ & 0.500 & 0.096 & \\
\hline \multicolumn{4}{|c|}{ Adjuvant treatment } \\
\hline ChT only & 22 & 12 & 0.418 \\
\hline ChT \& HT & 51 & 41 & \\
\hline $\mathrm{p}(\mathrm{uPA} / \mathrm{PAI} 1)^{*}$ & 0.806 & 1.000 & \\
\hline \multicolumn{4}{|l|}{ Tumor grade } \\
\hline Grade I & 4 & 6 & 0.434 \\
\hline Grade II & 34 & 21 & \\
\hline Grade III & 35 & 26 & \\
\hline $\mathrm{p}(\mathrm{uPA} / \mathrm{PAI} 1)^{*}$ & 0.425 & 0.395 & \\
\hline \multicolumn{4}{|l|}{ Tumor size } \\
\hline$\leq 20 \mathrm{~mm}$ & 22 & 14 & 0.693 \\
\hline$>20 \mathrm{~mm}$ & 51 & 39 & \\
\hline $\mathrm{p}(\mathrm{uPA} / \mathrm{PAI} 1)^{*}$ & 1.000 & 0.789 & \\
\hline \multicolumn{4}{|l|}{ HR status } \\
\hline Negative & 6 & 8 & 0.260 \\
\hline Positive & 67 & 45 & \\
\hline $\mathrm{p}(\mathrm{uPA} / \mathrm{PAI} 1)^{*}$ & 0.699 & 1.000 & \\
\hline Vascular invasior & & & \\
\hline
\end{tabular}

Table 2 continued

\begin{tabular}{llll}
\hline Characteristic & CMF & ANT & $P$ (treatment) \\
\hline No & 45 & 29 & 0.573 \\
Yes & 26 & 22 & \\
Unknown & 2 & 2 & \\
p(uPA/PAI1)* & 0.471 & 0.808 & \\
Lymph node status & & & 0.656 \\
Negative & 6 & 4 & \\
1-3 positive & 49 & 32 & \\
$>3$ positive & 18 & 17 & \\
p(uPA/PAI1)* & 0.880 & 0.973 & \\
\hline
\end{tabular}

* These $P$-values (uPA/PAI1) are for the characteristics grouped by treatment, e.g. comparing lymph node status for ANT-treated patients separately from CMF-treated patients with respect to uPA/PAI-1 status

\section{Results}

The experimental design is as given in Fig. 1. The median follow-up in the cohort of patients under observation was 37 months (range 1-84 months). Either local (just in one single case) or distant disease recurrence was observed in 49 out of 180 patients; 31 in the CMF arm and 18 in the anthracycline arm.

Survival data

The 3-year DFS was $86.0 \%$ in the group of 54 patients with both factors low, and $78.7 \%$ in the group of 126 patients with one or both factors high (Fig. 2); the difference did not reach the level of significance $(P=0.157)$. With respect to treatment groups, the 3-year DFS rates in the CMF group of 100 patients and in anthracycline group of 80 patients were similar, 79.7 and $82.6 \%$, respectively $(P=0.390)$ (Fig. 3).

\section{$\mathrm{CMF}$ arm}

In the group of 100 patients treated with CMF, a significant difference in DFS with respect to UPA and PAI-1 status was found (Fig. 4). The 3-year DFS was $87.1 \%$ in the low group $(n=27)$ and $77.0 \%$ in the high group $(n=73)$ $(P=0.044 ; \mathrm{HR}=2.81 ; 95 \% \mathrm{CI}=0.98-8.04)$.

Anthracycline arm

In the group of 80 patients treated with anthracycline-based chemotherapy, the difference in DFS with respect to uPA and PAI-1 disappeared (Fig. 5). The 3-year DFS was $85.2 \%$ in the low group $(n=27)$ and $81.8 \%$ in the high group $(n=53)$ $(P=0.807 ; \mathrm{HR}=0.88 ; 95 \% \mathrm{CI}=0.33-2.40)$. 


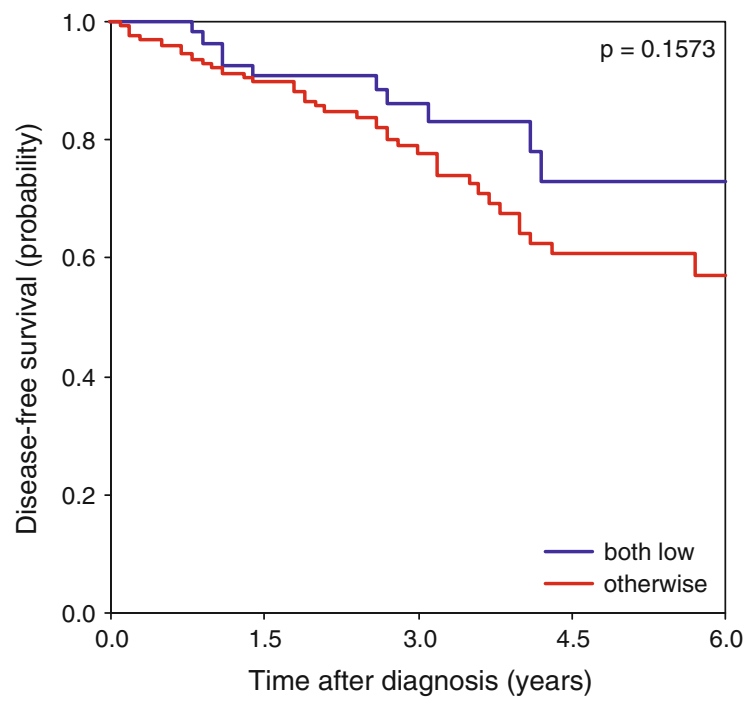

At risk:

$\begin{array}{rrrrrr}\text { both low } & 54 & 48 & 31 & 13 & 4 \\ \text { otherwise } & 126 & 111 & 70 & 33 & 13\end{array}$

Fig. 2 DFS for the whole cohort of patients according to uPA and PAI-1 levels $(N=180)$

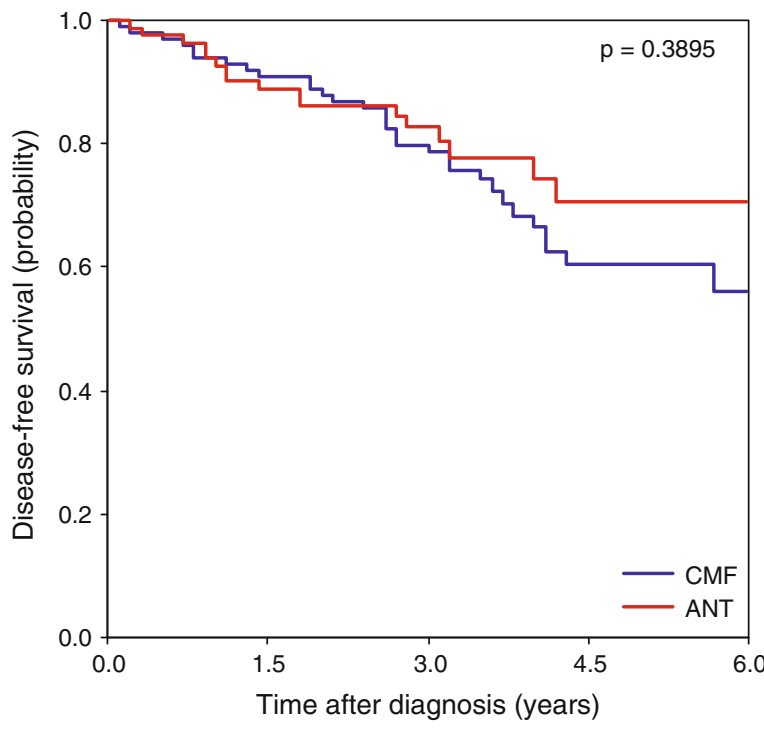

At risk:

$\begin{array}{lccccc}\text { CMF } & 100 & 89 & 61 & 30 & 11 \\ \text { ANT } & 80 & 70 & 40 & 16 & 6\end{array}$

Fig. 3 DFS for the whole cohort of patients according to treatment $(N=180)$

Low versus high levels of uPA and PAI-1

Patients with low levels of uPA and PAI-1 exhibited similar 3-year DFS rates regardless of treatment: $87.1 \%$ in the CMF group of 27 patients and $85.1 \%$ in the anthracycline group of 27 patients $(P=0.334)$. On the other hand,

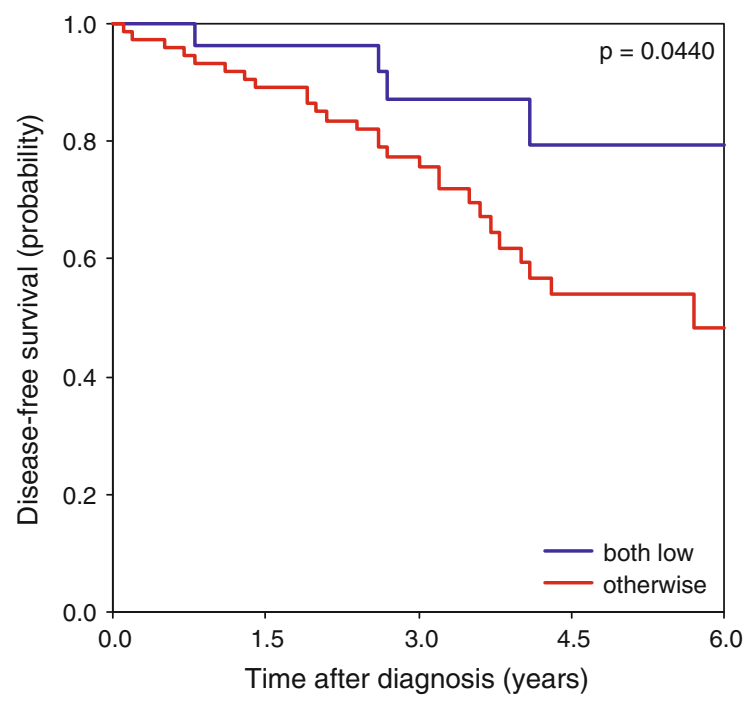

At risk:

$\begin{array}{rlllll}\text { both low } & 27 & 25 & 18 & 10 & 3 \\ \text { otherwise } & 73 & 64 & 43 & 20 & 8\end{array}$

Fig. 4 DFS in the CMF-treated patients according to uPA and PAI-1 levels $(N=100)$

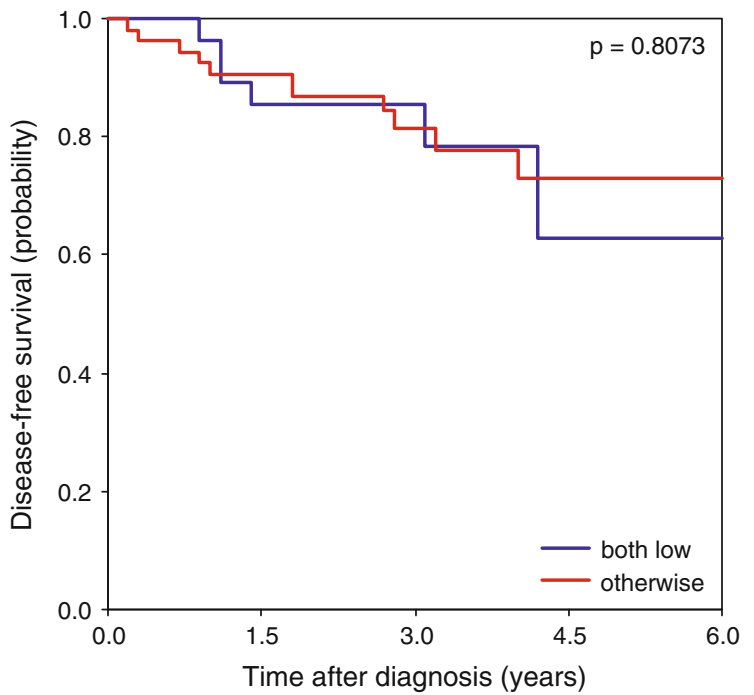

At risk:

$\begin{array}{rrrrcr}\text { both low } & 27 & 23 & 13 & 3 & 1 \\ \text { otherwise } & 53 & 47 & 27 & 13 & 5\end{array}$

Fig. 5 DFS in the anthracycline-treated patients according to uPA and PAI-1 levels $(N=80)$

patients with high uPA and/or PAI-1 levels revealed a trend towards better survival when treated with anthracyclines instead of CMF; the curves split after one and a half years of follow-up. The 3-year DFS was $77.0 \%$ in the CMF group of 73 patients and $81.1 \%$ in the anthracycline group of 53 patients $(P=0.147)$. 


\section{Discussion}

In breast cancer, markers that predict sensitivity of tumors to a particular systemic therapy are necessary to support the selection of the most optimal adjuvant treatment for each individual breast cancer patient since more precise selection of systemic therapy can improve outcomes and reduce adverse effects. Currently, hormone receptor status and HER-2 status are the only established factors predicting responsiveness to hormonal and HER-2 targeted therapy. The components of the plasminogen activator system uPA and PAI-1 are the only novel tumor biological factors that have reached level-1 evidence with regard to their prognostic impact in primary breast cancer. There is no doubt that at least the patients with high protease levels need some form of systemic therapy. However, there still exists a lack of data on whether UPA and/or PAI-1 levels can also serve as predictive markers for response to a particular systemic therapy, thus supporting the selection of the most appropriate systemic therapy for each individual patient.

The positive predictive value of the plasminogen activator system for response to adjuvant chemotherapy, mostly CMF, was already found in two prior retrospective observations $[18,20]$. Additionally, the Chemo N0 trial showed that nodenegative breast cancer patients with high uPA/PAI-1 levels benefit significantly from adjuvant chemotherapy with $\mathrm{CMF}$ $[13,14]$. In the ongoing NNBC-3 trial, optimal chemotherapy (anthracycline-taxane versus anthracycline-based) for patients with high uPA/PAI-1 levels is being evaluated and the results are eagerly awaited [26].

This paper is the first to show a difference in response to the anthracycline-containing regimen compared to response to CMF with respect to uPA and PAI-1 status in primary tumors. We found that patients with high uPA and/ or PAI-1 levels in primary tumor may benefit more from adjuvant anthracycline-containing regimens than from CMF. Our preliminary data based on the complete data set containing 1,220 patients of which the majority had been treated with some kind of adjuvant systemic therapy already pointed to the possible predictive value of uPA/ PAI-1 levels for response to anthracycline-based chemotherapy [21]. Since the selection of the type of adjuvant chemotherapy was based on the institutional guidelines valid at the time, the standard prognostic factors in two treatment groups were not balanced; the patients treated with anthracyclines tended to have anatomically more widespread disease, higher tumor grades and higher rates of vascular invasion that might have influenced our preliminary results. To avoid the unevenness in tumor characteristics, a further step with balancing the treatment groups was called for. With the balancing algorithm described in this paper we reached a well-balanced distribution of 180 patients in four groups determined by CMF and anthracycline-based chemotherapy and uPA/PAI-1 status. The algorithm in a way created a similar setting to that of a randomized study at the expense of greatly reducing the number of patients included into the study itself. In this setting, we observed that the 3-year DFS in the CMF arm differs significantly between patients with low and patients with high levels of markers $(87.1 \%$ vs. $77.0 \%, P=0.044$ ), while, on the other hand, the 3-year DFS in the anthracycline arm did not differ much between the two marker level subgroups $(85.2 \%$ vs. $81.8 \%$, $P=0.807$ ). Our observations show that worse prognosis correlated to high uPA and PAI-1 levels can be reversed by treatment efficacy achieved through anthracycline-based chemotherapy. This points to a possible predictive value of uPA/PAI-1 for sensitivity to anthracyclines. The situation is graphically represented in Fig. 6 and corresponds to "type 4 marker" as defined by Hamilton and Piccart [19]. Parallels can be drawn to a similar situation with HER-2 and trastuzumab, where same as in our case worse prognosis correlates with treatment efficacy.

It must be noted that the distributions of different characteristics after the balancing are not like those usually encountered in clinical settings with, for example, approximately half of the patients being node-negative; most of the patients were lymph node-positive in our selected subgroups. This was due to the fact that only very few lymph node-negative patients were treated with anthracycline-containing therapy at the time. However, there is no reason to believe that our observations made in the cohort of mostly node-positive early breast cancer patients cannot be extended to node negative disease. To confirm our observations, however, a prospective randomized trial comparing the efficacy of anthracyline and non-anthracycline-based chemotherapy in different uPA/

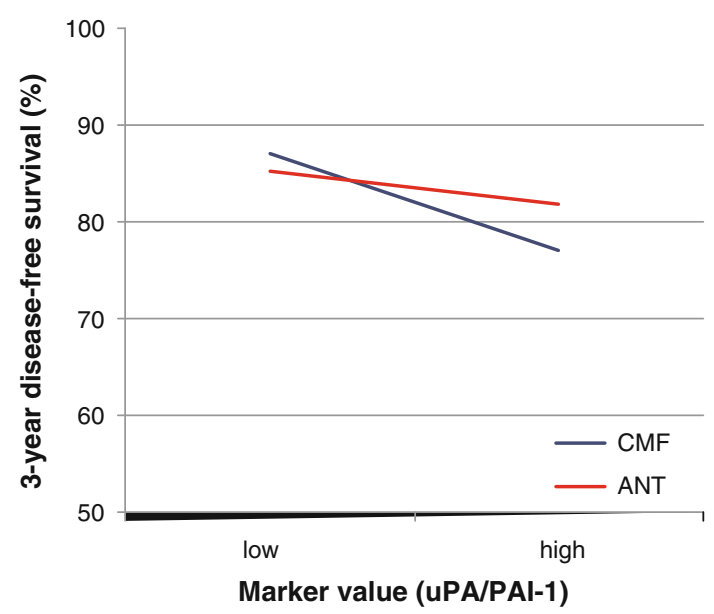

Fig. 6 Three-year DFS according to uPA/PAI-1 categories. This graphical representation shows parallels to the one introduced by Hamilton and Piccart [19] for "type 4 marker" 
PAI-1 subgroups of patients is needed. On the other hand, there is the question of whether our results can be applicable to different molecular subgroups of patients, as classified by the HR and HER-2 status. Unfortunately, due to the small groups of patients and a lack of routine HER-2 determination at the time of our study, no data on the influence of uPA/PAI-1 levels on response to different chemotherapeutic schedules in various molecular subtypes of early breast cancer can be obtained from our work.

HER-2 status has already been confirmed as a reliable predictive marker for response to anthracyclines in early breast cancer [2], and topoisomerase II $\alpha$ gene/protein co-expression, which is frequently found in HER-2 positive tumors and represents the major target for anthracyclines, was found to add some additional information [4, 27]. There is the question of whether uPA/PAI-1 levels, influencing stroma and cancer cells microinvasion, can add any additional independent information on anthracycline-based chemotherapy sensitivity in molecular subtypes defined by HER-2 status. Only limited data are available on the correlation between HER-2 status and uPA and/or PAI-1 levels in primary breast tumors. In our observation, performed on a cohort of 308 early breast cancer patients treated between the years 2004 and 2006 at the Maribor Teaching Hospital, Slovenia, a weak correlation between HER-2 status and uPA levels, but not PAI-1 levels, was found [28]. A French group also found a weak positive correlation between uPA/PAI-1 and HER-2 [29]. The study by Urban et al. indicates that overexpression of uPA mRNA levels in HER-2 positive breast cancer determines a worse outcome of the disease [30]. On the other hand, Harbeck et al. demonstrated that uPA/PAI-1 and HER-2 gave independent information on disease-free survival in lymph node-negative breast cancer patients [31] with a similar finding described by Konecny et al. [32] and by Zemzuoum et al. [33]. Therefore, uPA and PAI-1 levels might, independently of HER-2 status, help us to better select patients that will benefit from anthracyclines. According to a meta-analysis, there is the question of whether HER-2 negative patients do benefit from anthracyclines at all; however, a precise look at the pooled data, analyzed by Gennari et al., tells us that in the trials not so extensively contaminated by endocrine-responsive, luminal-type cancers, namely the Danish and NSABP B 11 trials, the benefit of anthracyclines over non-anthracycline schemes was observed also in HER-2 negative disease as well. This gives us confidence that even among HER-2 negative early breast cancer patients, there is a subset of patients that might benefit from anthracyclines. There is therefore an urgent need to identify the predictive markers that will help us to select such patients. Given that the methodology for uPA/PAI-1 determination is simple, validated and quality assured, measuring the uPA/PAI-1 levels might be another valuable marker for determining anthracycline sensitivity in the future.

In conclusion, the present paper confirms our previous observation that worse prognosis correlated with high uPA and PAI-1 levels can be reversed with improved treatment efficacy achieved by anthracycline-based chemotherapy, and not by CMF, which points to a possible predictive value of uPA/PAI-1 for sensitivity to anthracyclines. Patients with high uPA and/or PAI-1 levels in the primary tumor may benefit more from the adjuvant anthracyclinecontaining regimens than from CMF. The clinical application of the present work is to offer additional information that might facilitate the selection of the most appropriate candidates for anthracycline-based chemotherapy. However, for any firm conclusions on the predictive value of uPA/PAI-1 levels for the response to different systemic therapies in various molecular subtypes of early breast cancers, large prospective translational research trials are required.

\section{References}

1. Early Breast Cancer Trialists Collaborative Group (2005) Effects of chemotherapy and hormonal therapy for early breast cancer on recurrence and 15-year survival: an overview of the randomised trials. Lancet 365:1687-1717

2. Gennari A, Sormani MP, Pronzato P, Puntoni M, Colozza M, Pfeffer U, Bruzzi P (2008) HER2 status and efficacy of adjuvant anthracyclines in early breast cancer: a pooled analysis of randomized trials. J Natl Cancer Inst 100(1):14-20

3. Pritchard KI, Messersmith H, Elavathil L, Trudeau M, O'Malley F, Dhesy-Thind B (2008) HER-2 and topoisomerase II as predictors of response to chemotherapy. J Clin Oncol 26(5):736-744

4. O'Malley FP, Chia S, Tu D, Shepherd LE, Levine MN, Huntsman D, Bramwell VH, Andrulis I, Pritchard KI (2006) Prognostic and predictive value of topoisomerase II alpha in a randomized trial comparing CMF to CEF in premenopausal women with node positive breast cancer (NCIC CTG MA. 5). J Clin Oncol 24:11s (suppl; abstr 533)

5. Andreasen PA, Kjøller L, Christensen L, Duffy MJ (1997) The urokinase-type plasminogen activator system in cancer metastasis: a review. Int J Cancer 72:1-22

6. Schmitt M, Harbeck N, Thomssen C, Wilhelm O, Magdolen V, Reuning U, Ulm K, Höfler H, Jänicke F, Graeff H (1997) Clinical impact of the plasminogen activation system in tumor invasion and metastases: prognostic relevance and target for therapy. Tromb Haemostasis 78:285-296

7. Duffy MJ, Maguire T, McDermott EW, O'Higgins N (1999) Urokinase plasminogen activator: a prognostic marker in multiple types of cancer. J Surg Oncol 71:130-135

8. Look MP, Foekens JA (2000) Clinical relevance of the urokinase plasminogen activator system in breast cancer. APMIS 107:150 159

9. Borstnar S, Vrhovec I, Cufer T (2002) Prognostic value of plasminogen activator inhibitors in breast cancer. Int $\mathrm{J}$ Biol Markers 17(2):96-103

10. Borstnar S, Vrhovec I, Svetic B, Cufer T (2002) Prognostic value of the urokinase-type plasminogen activator, and its inhibitors 
and receptor in breast cancer patients. Clin Breast Cancer 3(2):138-146

11. Cufer T, Borstnar S, Vrhovec I (2003) Prognostic and predictive value of the urokinase-type plasminogen activator (uPA) and its inhibitors PAI-1 and PAI-2 in operable breast cancer. Int J Biol Markers 18(2):106-115

12. Look MP, van Putten WL, Duffy MJ, Harbeck N, Christensen IJ, Thomssen C, Kates R, Spyratos F, Fernö M, Eppenberger-Castori S, Sweep CG, Ulm K, Peyrat JP, Martin PM, Magdelenat H, Brünner N, Duggan C, Lisboa BW, Bendahl PO, Quillien V, Daver A, Ricolleau G, Meijer-van Gelder ME, Manders P, Fiets WE, Blankenstein MA, Broët P, Romain S, Daxenbichler G, Windbichler G, Cufer T, Borstnar S, Kueng W, Beex LV, Klijn JG, O'Higgins N, Eppenberger U, Jänicke F, Schmitt M, Foekens JA (2002) Pooled analysis of prognostic impact of urokinase-type plasminogen activator and its inhibitor PAI-1 in 8377 breast cancer patients. J Natl Cancer Inst 94(2):116-128

13. Jänicke F, Prechtl A, Thomssen C, Harbeck N, Meisner C, Untch M, Sweep CG, Selbmann HK, Graeff H, Schmitt M, German N0 Study Group (2001) Randomized adjuvant chemotherapy trial in high-risk, lymph node-negative breast cancer patients identified by urokinase-type plasminogen activator and plasminogen activator inhibitor type 1. J Natl Cancer Inst 93:913-920

14. Harbeck N, Schmitt M, Meisner C, Friedel C, Untch M, Schmidt M, Lisboa B, Sweep C, Jänicke F, Thomssen C, Chemo N0 Study Group (2009) Final 10-year analysis of prospective multicenter Chemo N0 trial for validation of ASCO-recommended biomarkers uPA/PAI-1 for therapy decision making in node-negative breast cancer. J Clin Oncol 27:15s (suppl; abstr 511)

15. Harbeck N, Alt U, Berger U, Krüger A, Thomssen C, Jänicke F, Höfler H, Kates RE, Schmitt M (2001) Prognostic impact of proteolytic factors (UPA, PAI-1, cathepsins B, D, L) in primary breast cancer reflects effects of adjuvant systemic therapy. Clin Cancer Res 7:2757-2764

16. Harbeck N, Kates R, Schmitt M (2002) Clinical relevance of invasion factors $\mathrm{UPA}$ and PAI-1 for individualized therapy decisions in primary breast cancer is greatest when used in combination. J Clin Oncol 20(4):1000-1007

17. Fernö M, Bendahl PO, Borg $\AA$, Brundell J, Hirschberg L, Olsson H, Killander D (1996) Urokinase plasminogen activator in breast cancer, analysed in steroid receptors cytosols with a luminometric immunoassay. Eur J Cancer 32A:793-801

18. Manders P, Tjan-Heijnen VCG, Span PN, Grebenchtchikov N, Geurts-Moespot A, van Tienoven DT, Beex LV, Sweep FC (2004) Predictive impact of urokinase-type plasminogen activator: plasminogen activator inhibitor type-1 complex on the efficacy of adjuvant systemic therapy in primary breast cancer. Cancer Res 64:659-664

19. Hamilton A, Piccart M (2000) The contribution of molecular markers to the prediction of response in the treatment of breast cancer: a review of the literature on HER-2, p53 and BCL-2. Ann Oncol 11:647-663

20. Harbeck N, Kates RE, Look MP, Meijer-Van Gelder ME, Klijn JG, Krüger A, Kiechle M, Jänicke F, Schmitt M, Foekens JA (2002) Enhanced benefit from adjuvant systemic chemotherapy in breast cancer patients classified high-risk according to urokinasetype plasminogen activator (uPA) and plasminogen activator inhibitor type-1 (PAI-1). Cancer Res 62:4617-4622

21. Borstnar S, Vrhovec I, Cufer T (2004) High levels of uPA and PAI-1 predicts a good response to antracyclines (abstract). Breast Cancer Res Treat 88(suppl. 1):54-55
22. McShane LM, Altman DG, Sauerbrei W, Taube SE, Gion M, Clark GM, Statistics Subcommittee of the NCI-EORTC Working Group on Cancer Diagnostics (2005) Reporting recommendations for tumor marker prognostic studies. J Clin Oncol 23(36):90679072

23. Elston CW (1987) Grading of invasive carcinoma of the breast. In: Page DL, Anderson TJ (eds) Diagnostic histopathology of the breast. Churchill Livingstone, Edinburgh, pp 300-311

24. Sweep CG, Geurts-Moespot J, Grebenschikov N, de Witte JH, Heuvel JJ, Schmitt M, Duffy MJ, Jänicke F, Kramer MD, Foekens JA, Brünner N, Brugal G, Pedersen AN, Benraad TJ (1998) External quality assessment of trans-European multicentre antigen determinations (enzyme-linked immunoabsorbent assay) of urokinase-type plasminogen activator (Upa) and its type 1 inhibitor (PAI-1) in human breast cancer tissue extracts. Br J Cancer 78:1434-1441

25. Gu XS, Rosenbaum PR (1993) Comparison of multivariate matching methods: structures, distances, and algorithms. J Comput Graph Stat 2(4):405-420

26. Annecke K, Schmitt M, Euler U, Zerm M, Paepke D, Paepke S, von Minckwitz G, Thomssen C, Harbeck N (2008) uPA and PAI1 in breast cancer: review of their clinical utility and current validation in the prospective NNBC-3 trial. Adv Clin Chem 45:31-45

27. Knoop AS, Knudsen H, Balslev E, Rasmussen BB, Overgaard J, Nielsen KV, Schonau A, Gunnarsdóttir K, Olsen KE, Mouridsen H, Ejlertsen B, Danish Breast Cancer Cooperative Group (2006) Retrospective analysis of topoisomerase IIa amplifications and deletions as predictive markers in primary breast cancer patients randomly assigned to cyclophosphamide, methotrexate, and fluorouracil or cyclophosphamide, epirubicin, and fluorouracil: Danish Breast Cancer Cooperative Group. J Clin Oncol 24(6): 1015

28. Ravnik M, Takac I, Arko D, Gorisek B, Cas-Sikosek N, Lampelj M, Ravnik J, Cufer T (2007) Correlation between invasive markers (uPA/PAI-1) and HER-2 status in early breast cancer. In: Samija M (ed). 5th Central European oncology congress, Opatija, Croatia, June 20-23. Final program \& abstract book: Croatian Oncological Society, p 81

29. Bouchet C, Ferrero-Poüs M, Hacene K, Becette V, Spyratos F (2003) Limited prognostic value of c-erbB-2 compared to uPA and PAI-1 in primary breast carcinoma. Int J Biol Markers 18(3): 207-217

30. Urban P, Vuaroqueaux V, Labuhn M, Delorenzi M, Wirapati P, Wight E, Senn HJ, Benz C, Eppenberger U, Eppenberger-Castori $S$ (2006) Increased expression of urokinase-type plasminogen activator mRNA determines adverse prognosis in ErbB2-positive primary breast cancer. J Clin Oncol 24(26):4245-4253

31. Harbeck N, Ross J, Yurdseven S, Dettmar P, Pölcher M, Kuhn W, Ulm K, Graeff H, Schmitt M (1999) HER-2/neu gene amplification determined by in-situ hybridization (FISH) allows risk group assessment in node-negative breast cancer. Int $\mathrm{J}$ Oncol 14:663-671

32. Konecny G, Untch M, Arboleda J, Wilson C, Kahlert S, Boettcher B, Felber M, Beryt M, Lude S, Hepp H, Slamon D, Pegram M (2001) HER-2/neu and urokinase-type plasminogen activator and its inhibitor in breast cancer. Clin Cancer Res 7:2448-2457

33. Zemzoum I, Kates RE, Ross JS, Dettmar P, Dutta M, Henrichs C, Yurdseven S, Höfler H, Kiechle M, Schmitt M, Harbeck N (2003) Invasion factors uPA/PAI-1 and HER2 status provide independent and complementary information on patients outcome in node-negative breast cancer. J Clin Oncol 21(6):1022-1028 